\title{
Epidemiology of Tuberculosis in Ardabil Province, 2011-2016
}

\author{
Firouz Amani ${ }^{1}$, Anahita Zakeri ${ }^{2 *}$, Mahin Sadeghnejad ${ }^{3}$, Vahid Abbasi ${ }^{4}$ and Jafar Mohammadshahi ${ }^{5}$ \\ 'Department of Community Medicine, Faculty of Medicine, Ardabil University of Medical Science, \\ Ardabil, Iran; firouz.amani@arums.ac.ir \\ ${ }^{2}$ Department of Internal Medicine, Faculty of Medicine, Ardabil University of Medical Science, \\ Ardabil, Iran; a.zakeri@arums.ac.ir \\ ${ }^{3}$ Ardabil TB Register Center, Ardabil University of Medical Science, Ardabil, Iran; msadeg75@yahoo.com \\ ${ }^{4}$ Department of Neurology, Faculty of Medicine, Ardabil University of Medical Science, \\ Ardabil, Iran.v.abbasi@arums.ac.ir \\ ${ }^{5}$ Department of Infection Disease, Faculty of Medicine, Ardabil University of Medical Science, \\ Ardabil, Iran; j.mohammadshahi@arums.ac.ir
}

\begin{abstract}
Background: Tuberculosis (TB) is a chronic bacterial disease caused by mycobacterium tuberculosis that appears in $85 \%$ of cases by pulmonary form. Epidemiologic study of disease during the time can have important rule for evaluation strategy of diseases control and trend of health indices. Purpose: The aim of this study was to investigate the epidemiology of Tuberculosis in Ardabil province. Method: This descriptive cross-sectional retrospective study was conducted on 797 TB patients who referred to health centers in Ardabil province. Information was collected from patients' records and then analyzed by statistical methods in SPSS version 19. Results: The average age of patients was $44.3 \pm 18.1$ (1-91 years). 46\% of patients were male and the rest were female. $83 \%$ of patients were living in urban and $18 \%$ in rural areas. The mean of total incidence of TB in Ardabil province during study years was 10.26 per 100000 . From all TB cases, $56.71 \%$ was pulmonary and rest was extra-pulmonary. The most common organs involved in extra-pulmonary TB were lymph nodes with $24.21 \%$. Conclusion: More considering to the early known of Pulmonary $\mathrm{Tb}$ and starting treatment after confirmation and also considering to the lower age of TB cases, aassessment the barriers to implementation of control, therapeutic and educational programs about TB cases is essential.
\end{abstract}

Keywords: Ardabil, Epidemiology, Incidence, Tuberculosis

\section{Introduction}

Tuberculosis (TB) is a common cause of death due to Single-agent infectious diseases in world and despite the advances in medical science; it remains a health problem in many countries. TB is occurring due to Mycobacterium Basil and usually enters to body during infancy. By over time and also due to weakening of the immune system, Bacilli were multiplying and cause recurrence of the disease. TB had the $10^{\text {th }}$ rank in burden of diseases and it predicted that till 020 it reaches to $7^{\text {th }}$ rank.
According to the report of WHO in year 2011 the total cases of TB was 14.8 million and yearly about 9.3 million suffer to TB and 18 million die due to TB.

According the report of Iran ministry health, yearly 14.4 cases per 100000 suffered to TB. The incidence of extra-pulmonary in Iran from 2011 with 4.16 per 100000 decreased and reaches to 3.2 per 100000 in 2016 (2555 cases). About one-third of people with AIDS are associated with tuberculosis and this rate in Mediterranean was $23 \%$. The HIV infection increase the risk of TB activation more than 10 times and according to the WHO in year 
2011 about 13\% of TB patients also had HIV. Tb could infect whole organs of the body, but the common form of TB is pulmonary that in adults, it is usually accompanied with smear positive sputum and highly contagious. TB appears in $85 \%$ of cases by pulmonary form and rest of them by extra-pulmonary form. Khazaei et al., in a study on $440 \mathrm{~TB}$ patients with smear positive sputum found that the median time for negative result of smear sputum in patients was 3 months. In the end of second month, smear sputum in $69 \%$ of patients was negative and this rate reach to $88 \%$ in the end of third month ${ }^{1}$.

One of the Millennium Development Goals (MDGs), was decreasing the rate of death due to TB in 2015 to half of 1990 death rate. Because of Iran's neighbors with Pakistan and Afghanistan countries and also due to the higher rate of TB in these countries, the risk of suffering to TB in our country is high and Iran had the $17^{\text {th }}$ rank in the world ${ }^{2}$.

Gupta et al., in a study found that factors such as smoking, Alcohol consumption, liver and kidney diseases, mall-nutrition and history of TB in family are effective factors ${ }^{3}$.

Heshmati et al., in a study showed that $74.7 \%$ of TB patients are in the form of pulmonary and most of extrapulmonary cases were lymph nodes with $3.3 \%{ }^{4}$.

Shaghafipour et al., in a study during 2003-2010 found that $71.9 \%$ of pulmonary $\mathrm{TB}$ cases were positive smear and rest of the negative smear ${ }^{5}$.

In a study done by Hazrati et al., in Ardabil in 20052010 results showed that the TB total incidence rate was 8.54 per 100000 in Ardabil city and 56.2\% of patients have pulmonary TB and rest of them have extra-pulmonary $\mathrm{TB}^{6}$.

Salek et al., in a study showed that the incidence of positive smear pulmonary TB decreased 4 times and also the rate of other TB cases decreased. Until age 10 year, there wasn't difference between two sexes but in age groups 10-14, the rate of incidence in girls was 2 times boys. Also, they found that this reduction can be related to improve the care system and health promotion?

Beyramvand et al., in a study in 2014 showed that the simultaneous incidence of HIV infection and tuberculosis in the southwest of Iran was high ${ }^{2}$.

Study the epidemiology of TB during time and identify of disease trends and its changes in over time can be great importance in implications and achieve to using strategies for control disease, improve health and health promotion. The aim of this study was to investigate the epidemiological situation of tuberculosis in Ardabil province during 2011-16.

\section{Materials and Methods}

This retrospective descriptive cross-sectional study has been done on all $797 \mathrm{~TB}$ patients that registered in Ardabil province from 2011 to 2016 . The sampling method was census and all $\mathrm{TB}$ registered cases during study years entered the study and all TB cases confirmed by experimental tests by specialists. All patients with positive smear results entered the study and doubtful cases without TB excluded from the study. Personal Information and epidemiological data such as age, Sex, residence place, name of city and clinical data (Smear results, type of $\mathrm{TB}$, and cause of extra-pulmonary $\mathrm{TB}$ ) were collected by a checklist for all registered TB patients and then analyzed through SPSS.16 software by descriptive and analytical statistical methods. Also, we used the population statistics of each study years for estimation the incidence of TB in Ardabil province during the study years. The data extracted from the national TB registered data of Ardabil province which ethically approved by Ardabil University of Medical Science, Ardabil, Iran.

\section{Results}

Of all patients, 429 (54\%) cases were male and rest of them was female. The mean age of patients was $44.3 \pm 18.1$ in ranging 1 to 91 years. 662 (83\%) of patients were living in urban and $17 \%$ in rural areas. (Table 1 )

The most of $\mathrm{Tb}$ cases were seen in age group 35-65 years and the least of cases were in age group low than 10 years. The rate of incidence of TB during study years had swinging (Table 2).

During study years, 452 cases (56.7\%) had pulmonary TB that of them $52.2 \%$ occurred among men and $63.1 \%$ among urban people. There wasn't significant relation between TB type and sex but the relation between type of $\mathrm{TB}$ and residence place was significant (Table 3 ).

Of all cases, $0.6 \%$ had HIV, $56.7 \%$ had pulmonary TB and of them $77.2 \%$ had positive smear results (Table 4).

$2.3 \%$ of patients had a prison history. The mean of overall TB incidence rate was 9.3 per hundred thousand in the city of Ardabil during the study years (Table 1). 
Table 1. Demographic characterized of patients by year

\begin{tabular}{|c|c|c|c|c|c|c|c|c|c|c|}
\hline \multirow{3}{*}{ year } & \multicolumn{4}{|c|}{ Residence place } & \multicolumn{4}{|c|}{ Sex } & \multirow{3}{*}{$\begin{array}{c}\text { Total } \\
\text { number }\end{array}$} & \multirow{3}{*}{$\begin{array}{l}\text { Incidence rate per one } \\
\text { hundred thousand }\end{array}$} \\
\hline & \multicolumn{2}{|c|}{ Urban } & \multicolumn{2}{|c|}{ Rural } & \multicolumn{3}{|c|}{$\mathrm{f}$} & \multirow{2}{*}{$\begin{array}{l}\mathrm{m} \\
\%\end{array}$} & & \\
\hline & $\mathrm{n}$ & $\%$ & $\mathrm{n}$ & $\%$ & $\mathrm{n}$ & $\%$ & $\mathrm{n}$ & & & \\
\hline 2011 & 89 & 64.1 & 50 & 35.9 & 78 & 56.1 & 43.8 & 43.8 & 139 & 10.9 \\
\hline 2012 & 106 & 63 & 62 & 37 & 91 & 54.1 & 45.8 & 45.8 & 168 & 13.2 \\
\hline 2013 & 88 & 61.1 & 56 & 38.9 & 65 & 45.1 & 54.8 & 54.8 & 144 & 11.2 \\
\hline 2014 & 67 & 53.6 & 58 & 46.4 & 71 & 56.8 & 43.2 & 43.2 & 125 & 9.5 \\
\hline 2015 & 81 & 66.9 & 40 & 33.1 & 71 & 58.6 & 41.3 & 41.3 & 121 & 9.1 \\
\hline 2016 & 72 & 72 & 28 & 28 & 53 & 53 & 47 & 47 & 100 & 7.6 \\
\hline Total & 503 & 63.1 & 294 & 36.9 & 429 & 53.8 & 46.1 & 46.1 & 797 & 62.5 \\
\hline
\end{tabular}

Table 2. Frequency of TB cases by age groups

\begin{tabular}{llllll}
\hline Years & $<=10$ & $10-35$ & $35-65$ & $>65$ & Total \\
\hline 2011 & 0 & 51 & 65 & 23 & 139 \\
2012 & 4 & 58 & 73 & 33 & 168 \\
2013 & 5 & 50 & 61 & 28 & 144 \\
2014 & 3 & 41 & 61 & 20 & 125 \\
2015 & 3 & 26 & 63 & 29 & 121 \\
2016 & 1 & 30 & 57 & 12 & 100 \\
Total & 16 & 256 & 380 & 145 & 797 \\
\hline
\end{tabular}

$172(56.2 \%)$ of patients have TB in pulmonary form and others in extra pulmonary. From the organs involved in extra-pulmonary tuberculosis, most of them related to the lymph nodes with 35 cases (26.1\%) (Table 2).

According to the results, the total rate of TB in year 2012 with 13.2 per 100000 was the most (Table 5).

Of all extra-pulmonary TB cases, the most of TB cases related to lymph nodes Tuberculosis with 84 cases (24.2\%) (Table 6).

Table 3. Frequency of TB cases by sex and residence place and type of TB

\begin{tabular}{lcccccccccc}
\hline & \multicolumn{4}{c}{ Residence place } & \multicolumn{3}{c}{ Sex } \\
\cline { 2 - 9 } Type of TB & \multicolumn{2}{c}{ Urban } & \multicolumn{2}{c}{ Rural } & & Male & & Female \\
\cline { 2 - 9 } & $\mathrm{n}$ & $\%$ & $\mathrm{n}$ & $\%$ & $\mathrm{n}$ & $\%$ & $\mathrm{n}$ & $\%$ \\
\hline Pulmonary TB & 285 & 63.1 & 167 & 36.9 & 216 & 47.8 & 236 & 52.2 \\
Extra-Pulmonary TB & 197 & 57.1 & 148 & 42.8 & 213 & 61.7 & 132 & 38.3 \\
Total & 482 & 60.5 & 315 & 39.5 & 429 & 53.8 & 368 & 46.2 \\
\hline
\end{tabular}

Table 4. Frequency of patients by years, smear results and type of TB

\begin{tabular}{lcccccccc}
\hline \multirow{2}{*}{ years } & \multicolumn{2}{c}{ Positive smear } & \multicolumn{2}{c}{ Negative smear } & \multicolumn{2}{c}{ Total cases } & \multicolumn{2}{c}{ Extra-pulmonary cases } \\
\cline { 2 - 8 } & $\mathrm{n}$ & $\%$ & $\mathrm{n}$ & $\%$ & $\mathrm{n}$ & $\%$ & $\mathrm{n}$ & $\%$ \\
\hline 2011 & 67 & 48.2 & 9 & 0.06 & 76 & 54.7 & 63 & 45.3 \\
2012 & 89 & 53 & 22 & 13.1 & 111 & 66.1 & 57 & 33.9 \\
2013 & 63 & 43.8 & 28 & 19.4 & 91 & 63.2 & 53 & 36.8 \\
2014 & 56 & 44.8 & 13 & 10.4 & 69 & 55.2 & 56 & 44.8 \\
2015 & 42 & 34.7 & 18 & 15 & 60 & 50 & 61 \\
2016 & 32 & 32 & 13 & 13 & 45 & 45 & 55 & 50.4 \\
Total & 349 & 43.8 & 103 & 12.9 & 452 & 56.7 & 345 & 43.3 \\
\hline
\end{tabular}


Table 5. TB indicators during years 2011-2016

\begin{tabular}{|c|c|c|c|c|c|c|}
\hline Indicators & 2011 & 2012 & 2013 & 2014 & 2015 & 2016 \\
\hline Incidence of smear-positive pulmonary tuberculosis & 4.7 & 6.7 & 4.6 & 4.2 & 2.9 & 2.3 \\
\hline Incidence of smear-negative pulmonary tuberculosis & 0.4 & 1.3 & 1.8 & 0.9 & 1.3 & 0.8 \\
\hline Incidence of extra- pulmonary tuberculosis & 4.9 & 4.5 & 4.1 & 4.3 & 4.6 & 4.2 \\
\hline Total Incidence of tuberculosis & 10.9 & 13.2 & 11.2 & 9.5 & 9.2 & 7.6 \\
\hline Proportion of smear-positive TB to pulmonary tuberculosis & 67 & 89 & 63 & 59 & 42 & 32 \\
\hline smear-positive new cases & 83 & 107 & 72 & 79 & 48 & 44 \\
\hline Rate of recovery in patients with smear-positive pulmonary tuberculosis & 86.67 & 84.71 & 83.05 & 87.27 & 89.74 & 81.82 \\
\hline $\begin{array}{l}\text { Rate of successful treatment in patients with smear-positive pulmonary } \\
\text { tuberculosis }\end{array}$ & 91.67 & 88.24 & 83.05 & 89.09 & 89.74 & 81.82 \\
\hline
\end{tabular}

Table 6. Distribution of cases with extra-pulmonary tuberculosis

\begin{tabular}{lll}
\hline Extra pulmonary tuberculosis & Number & Percentage \\
\hline lymph nodes Tuberculosis & 84 & 24.21 \\
Pleural & 49 & 14.12 \\
Bone Tuberculosis & 59 & 17 \\
Tuberculosis Urinary & 19 & 5.48 \\
Billion & 8 & 2.31 \\
Pericardial & 4 & 1.15 \\
Tuberculosis meningitis & 7 & 2.02 \\
Digestion & 9 & 2.59 \\
skin TB & 11 & 3.17 \\
Peritoneal lining & 20 & 5.76 \\
Genital & 1 & 0.29 \\
Eye & 31 & 8.93 \\
Other & 23 & 6.63 \\
Total & 22 & 6 \\
\hline
\end{tabular}

Results showed that the rate of smear positive and negative TB after 2012 had decreasing trend till end of study (Figure 1).

\section{Discussion}

During study years, in Ardabil Province the mean of incidence rate was low than 11 per 100000 even though fluctuations have been observed over these six years. In Ebrahimzadeh et al., study the incidence rate of TB in Birjand had decreasing trend. Also, in Sofian study in

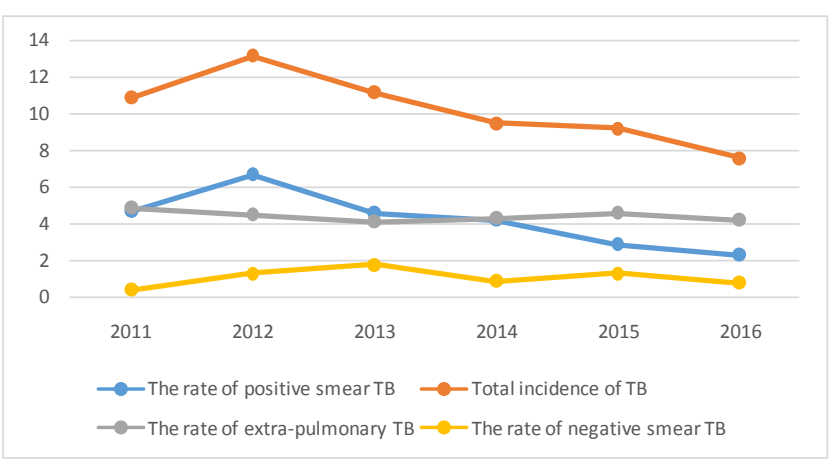

Figure 1. Changes trend of TB indices during study years.

Arak and Yazdani in Mazandaran there was decreasing trend. In Arsang study the total TB incidence rate had decreasing trend ${ }^{8-11}$

The mean age of patients in this study was 44 which was in line with Beyramvand et al., study in south-east of Iran with 39 year and Vosougi et al., study in Hamadan with 54 year $^{2,12}$.

In this study most of cases were in age group up 65 years. The most cases in Ilam and Arsang studies was up 65 years, in Hamadan up 50 year and in some places were in range $40-70$ years ${ }^{8,10-14}$.

As the age of the immune system increases, as the age grows, the chance of becoming a tuberculosis infection increases with tuberculosis and also, we can consider the increasing age as a risk factor for TB suffering. In this study $39.5 \%$ of cases were in rural and $60.5 \%$ in urban areas which in other studies in Iran the urban rate changes from $51 \%$ to $64 \%$ which was in line with our study results ${ }^{14-16}$.

The results showed that of all cases, 56.7\% had pulmonary TB and $43.3 \%$ had extra-pulmonary TB. Results showed that the type of $\mathrm{TB}$ in Ardabil province related 
to the TB status in other places. The most of smear positive pulmonary TB cases is related to 2012 year, after 2012 year was declining that statistically no significant, it can be concluded that number of patients found have a constant trend. From all 797 patients, $43.8 \%$ in smear positive pulmonary $\mathrm{TB}, 12.9 \%$ in smear negative pulmonary $\mathrm{TB}$ and $61.7 \%$ in extra pulmonary $\mathrm{TB}$ cases were men that is similar to other studies ${ }^{7,12}$. Tb is the second leading cause of death from an infectious disease worldwide (after HIV which caused an estimated 1.8 million deaths in 2008). Tuberculosis is the largest cause of death from single factor infectious diseases in the world (more than Aids, Malaria and Measles). TB is in the tenth rank of burden diseases and predicted until 2020 maintain its current position and some time increase to seven ranks. Currently, the incidence of smear positive pulmonary $\mathrm{TB}$ is the main indicator to measure the situation of $\mathrm{TB}$ in the community is the number of people with smears positive pulmonary TB during a year per hundred thousand people. Doing screening tests for exposed people, applied new diagnosis and treatment methods must be included in TB control strategic programs. Also achieve to the smear positive case identification more than $85 \%$ and success in TB treatment rate more than $90 \%$ is predicted in country of Iran FOR 2025. Obviously, the lower mean age of patients reflects the situation of TB control is worse and upper mean age reflect it is better. In this study $39.5 \%$ of patients were in urban area which is similar with references resource. Primarily in areas with high population density and poor, TB prevalence expected to be high.

Correct diagnosis of pulmonary tuberculosis is one of the problems in medicine and also as an important factor in TB epidemiology. In this study the frequency of patients in age group 35-65 years with $380(47.8 \%)$ is more than other age groups and based on the mean age of 44 years for patients can say that the pattern of disease in Ardabil city was slightly different from other places so, in other studies the disease is more in age up 60 years which this difference to became one of the challenges in the city of Ardabil ${ }^{7,8,12,14}$. In this study, pulmonary TB cases (56.7\%) were more than extra- pulmonary cases and also, the most common organ involved in extra-pulmonary TB was lymph with $24.2 \%$ which is similar to other provinces patterns $^{7,8,14,15}$. TB incidence rate in Iran has decreasing trend in recent ten years. The study on 134 countries in years 1997-2006 on TB incidence rate showed that the annually TB incidence decreasing rate is rapidly in high income countries ${ }^{15}$. According to statistics during the study years, the prevalence and incidence of TB in Iran has been decreased from 40 and 24 per 100000 in 2005 to 27 and 17 per 100000 in 2010; respectively. Also, TB incidence rate may be having significantly relation with welfare indicators such as income level, GDP and social conditions.

\section{Conclusion}

Due to the lack of decrease in the incidence of extra pulmonary tuberculosis in Ardabil province, we should have done more studies about this disease and effected causes for transmit, prediction and treatment of TB in Ardabil province. Also, by considering the affect of age on disease occurrence, it suggested that we most done exact screening in high risk age groups. Also, higher percentage of simultaneous HIV infections and tuberculosis in society and its effect on general health, so screening and early diagnosis of infections, training and doing necessary actions for prediction of people to suffering TB is recommended in future.

\section{Conflict of Interest}

None

\section{References}

1. Khazaei S, Kousehlou Z, Karami M, Zahiri A, Bathaei J. Time to sputum conversion among patients with smearpositive pulmonary tuberculosis and its determinants: A retrospective cohort study in Hamadan Province, Iran. Iranian Journal of Epidemiology. 2013; 9(1):32-40.

2. Beiranvand R, Ghalavandi S, Delpisheh A, Sayemiri K, Salmanzadeh S. Epidemiology of tuberculosis in south-west of Iran: A brief report. Tehran University Medical Journal. 2014; 72(4):263-7. (Persian)

3. Gupta S, Shenoy VP, Mukhopadhyay C, Bairy I, Muralidharan S. Role of risk factors and socio-economic status in pulmonary tuberculosis: A search for the root cause in patients in a tertiary care hospital, south India. Tropical Medicine International Health. 2011; 16(1):74-8. Crossref PMid:21091857

4. Heshmati H, Ravanbakhsh K, Khajavi S, Behnampour N. Epidemiologic study of tuberculosis in Galikesh city during 2006-2011. Iran J Med Microbiol. 2015; 9(1):61-5. 
5. Saghafipour A, Noroozei M, Mostafavi R, Heidarpour A, Ghorbani M. The epidemiologic status of Pulmonary tuberculosis and its associated risk factors in Qom province during 2002-2010. J Mazandaran Univ Med Sci. 2012; 22(90):63-70.

6. Hazrati S, Khaligh N, Moeini A, Amani F, Barak M, Rahimi $\mathrm{G}$ et al. Epidemiology of tuberculosis in Ardabil City from 2005 to 2010. J Health. 2013; 4(2):103-9.

7. Salek S, Masjedi M, Salek S, Emami H. Incidence rate of pulmonary tuberculosis among different ethnicities in Golestan Province from 1999 to 2003. IRJE. 2008; 3(3 and 4):15-20.

8. Ebrahimzadeh A, Sharifzadeh GR, Eshaghi S. The epidemiology of tuberculosis in Birjand (1996-2006). Journal of Birjand University of Medical Sciences. 2009; 16(1):31-8.

9. Charati JY, Kazemnejad A, Mosazadeh M. An epidemiological study on the reported cases of tuberculosis in Mazandaran (1999-2008) using spatial design. J Mazandaran Univ Med Sci. 2010; 19(74):9-16.

10. Sofian M, Zarinfar N, Mirzaee M, Nejad AM. Epidemiology of tuberculosis in Arak, Iran. Koomesh. 2009; 10(4):261-6.
11. Arsang S, Kazemnejad A, Amani F. Epidemiology of tuberculosis in Iran (2001-2008). Journal of Gorgan University of Medical Sciences. 2011; 13(3):86-7.

12. Vosogh RN, Roshanaei G, Khazaei S, Safari M, Zahiri A, Bothaei J. Study of tuberculosis epidemiology and its affected factors in Hamadan province, during the years 2007-2013. PSJ. 2015; 14(1):64-71.

13. Jamshidi K, Peyman H, Pakzad I, Delpisheh A. Trends and some risk factors for incidence of tuberculosis in Ilam province (Western Iran). Journal of Ilam University of Medical Science. 2011; 19(3):32-40.

14. Yousefnejad S, Mousavi-jarrahi A, Mehrabi Y, Hatami H. Epidemiolgy of pulmonary tuberculosis of smear positive in Kurdistan Province (Iran). Iranian Journal of Infectious Diseases and Tropical Medicine. 2013; 62:49-52.

15. Rahmanian V, Rahmanian K, Safary AR, Mansoorian E, Rastgoofard MA. The epidemiology of tuberculosis in Jahrom (2005-2014). JJUMS. 2016; 14(4):16-24. Crossref

16. Mohamadi Azni S, Mansourian AA, Nokandeh Z. Epidemiological study of tuberculosis in Damghan city (Iran) during 2003-2007. Koomesh. 2008; 9(4):315-20. 